\title{
CPR Artefact Removal from VF Signals by Means of an Adaptive Kalman Filter Using the Chest Compression Frequency as Reference Signal
}

\author{
S Ruiz de Gauna, J Ruiz, U Irusta, E Aramendi, A Lazkano, JJ Gutierrez \\ University of the Basque Country, Bilbao, Spain
}

\begin{abstract}
Chest compressions during Cardiopulmonary Resuscitation $(C P R)$ generate important artefacts which impede the correct analysis of the ECG signal by an automated external defibrillator (AED). The suppression of the CPR artefact is thus necessary during resuscitation.

The filtering technique proposed in the present paper is based on a Kalman adaptive scheme where the CPR artefact model follows the instantaneous frequency of the chest compressions, while the VF signal is modeled as a sum of two sinusoidal signals.

The good results obtained for the increase of the signal to noise ratio and the excellent sensitivity scores provided by a commercial AED on the cleaned VF signals certifies the value of the method.
\end{abstract}

\section{Introduction}

Early defibrillation is essential in the chain of survival; for every minute delay in its application the probability of restoration of spontaneous circulation falls by approximately $10 \%$. Automatic External Defibrillators (AED) are able to diagnose lethal ventricular arrhythmias, predominantly Ventricular Fibrillation (VF), and to consequently apply an electrical discharge. Survival rates of patients in cardiac arrest have therefore been substantially improved.

Current guidelines recommend cardiopulmonary resuscitation (CPR) before defibrillation. It has been shown that CPR prevents VF from converting to asystole, contributes to the sustained cebro-vascular function and increases the probability of a successful defibrillation [1].

Thoracic compressions (with or without ventilation) applied during CPR introduce a severe interference in the ECG signal which affects the reliability of the cardiac rhythm classification of AEDs [2]. The suppression of the CPR interference would allow AED rhythm analysis and CPR to simultaneously be performed, thus optimizing the resuscitation scenario.

The first studies on this field were conducted on pigs using mechanically applied CPR on induced VF rhythms. A high pass filter with fixed coefficients was successfully applied to eliminate the interference [3, 4]. Subsequent works, based on mixed human VF and animal CPR, concluded that adaptive techniques are more adequate [5, 6]. This is due to the spectral overlap of the human VF and the CPR interference and the high variability of the signals. These studies, as well as more recent works that use a complete human signal model [7], required the recording of up to four reference signals strongly correlated to the interference (i.e. compression depth, thoracic impedance, compression acceleration and ECG common mode). The difficulty associated to the recording of these signals in a life emergency situation is the fundamental limitation of the cited studies.

The solution proposed in this work is based on an adaptive Kalman filter using as a reference signal the instants corresponding to the thoracic compressions.

\section{Materials and methods}

\subsection{Data acquisition}

A controlled recording of a CPR artefact in a real emergency situation is very costly. There are however occasions were basic life support personnel arrive when the patient is already in asystole. If the AED initially detects a non shockable rhythm the CPR procedure is initiated. In such a case the AED directly records the CPR artefact over asystole. Following this procedure $17 \mathrm{CPR}$ registers have been obtained, showing a variable duration between 15 and 35 seconds.

The VF database used is composed of 191 records from diverse sources: recordings from the Cardiolab system, MIT/BIH-AHA commercial databases and digitalized paper registers from manual and automatic external defibrillator interventions. The duration of these records varies from 5 to 25 seconds.

Finally all the records were resampled at a $250 \mathrm{~Hz}$ sampling rate and filtered $(0.5-30 \mathrm{~Hz})$ to eliminate DC interferences, base line drifts and power line interferences. 


\subsection{Reference signal}

The studied filtering method uses the thoracic compression instants as a reference signal strongly correlated to the CPR interference.

Commercial AEDs, used by the emergency services to obtain the CPR registers, are not adequately prepared to record the reference signal. Consequently the CPR registers have been processed offline in order to mark the instants corresponding to the thoracic compressions, as shown in figure 1.

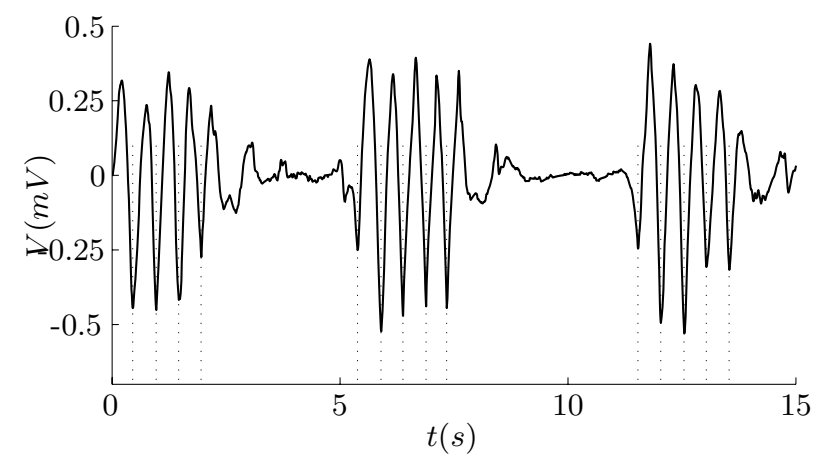

Figure 1. Reference signal for CPR11

\subsection{Test signal}

Test signals composed of a VF signal and a CPR interference with a known input signal to noise ratio $S N R_{i}$, are need to evaluate the goodness of the filtering technique. In the present study, the test signals have been constructed following the methodology established in [5]. A weighted CPR interference is added to the VF signal to obtained the desired $S N R_{i}$.

\subsection{The Kalman filter}

Adaptive Kalman filtering techniques have been applied in the present work to separate the CPR artefact from the VF signal. The Kalman filter is formulated using the statespace approach, in which a dynamical system is described by a set of variables called the state.

The instants corresponding to the thoracic compressions, the reference signal, is used to model the CPR interference. Calculating the mean value of the compression frequency $\left(f_{o} H z\right)$ is straightforward; the artefact is modeled as a sinusoidal signal of frequency $f_{o}$ and variable phase and amplitude as:

$$
\begin{aligned}
& s_{c p r}[n]=A_{0}[n] \cos \left(\Omega_{0} n+\theta_{0}[n]\right)= \\
& A_{0}[n] \cos \theta_{0}[n] \cos \Omega_{0} n-A_{0}[n] \sin \theta_{0}[n] \sin \Omega_{0} n
\end{aligned}
$$

where $\Omega_{0}$ is the discrete frequency corresponding to $f_{0}$. Let $x_{1}[n]=A_{0}[n] \cos \theta_{0}[n]$ and $x_{2}[n]=A_{0}[n] \sin \theta_{0}[n]$ be the state variables used to model the CPR interference, then:

$$
s_{c p r}[n]=\left\{\cos \Omega_{0} n,-\sin \Omega_{0} n\right\} \cdot\left\{\begin{array}{l}
x_{1}[n] \\
x_{2}[n]
\end{array}\right\}
$$

There is no a-priori information available to model the VF signal. Two sinusoidal signals of variable amplitude and phase and frequencies of 4 and $5 \mathrm{~Hz}$ produce an adequate model for the whole VF signal ensemble. The analytical description of the VF signal is:

$$
\begin{aligned}
s_{v f}[n]= & A_{1}[n] \cos \left(\Omega_{1} n+\theta_{1}[n]\right)+ \\
& A_{2}[n] \cos \left(\Omega_{2} n+\theta_{2}[n]\right)
\end{aligned}
$$

where $\Omega_{1}$ and $\Omega_{2}$ are the discrete frequencies corresponding to $f_{1}=4 \mathrm{~Hz}$ and $f_{2}=5 \mathrm{~Hz}$.

The four state variables that model the VF signal are:

$$
\left\{\begin{array}{l}
x_{3}[n] \\
x_{4}[n] \\
x_{5}[n] \\
x_{6}[n]
\end{array}\right\}=\left\{\begin{array}{l}
A_{1}[n] \cos \theta_{1}[n] \\
A_{1}[n] \sin \theta_{1}[n] \\
A_{2}[n] \cos \theta_{2}[n] \\
A_{2}[n] \sin \theta_{2}[n]
\end{array}\right\}
$$

$\left(A_{1}[n], \theta_{1}[n]\right)$ represent the amplitude and phase of the $f_{1}$ component and $\left(A_{2}[n], \theta_{2}[n]\right)$ the ones corresponding to $f_{2}$.

The test signal, $s_{\text {test }}[n]$, is obtained by adding $s_{v f}[n]$ and $s_{c p r}[n]$.

Kalman adaptive filtering describes the system model through two equations:

- The process equation, which describes the state variables at instant $n+1$ from their value at instant $n$; in matrix notation:

$$
\mathbf{x}[n+1]=\mathbf{\Phi}[n+1, n] \mathbf{x}[n]+\mathbf{v}_{\mathbf{1}}[n]
$$

where $\mathbf{x}[n]_{M \times 1}$ represents the state variable vector at instant $n$ and $\boldsymbol{\Phi}[n+1, n]_{M \times M}$ the state transition matrix from instant $n$ to $n+1$. The $\mathbf{v}_{\mathbf{1}}[n]_{M \times 1}$ vector represents a white noise process with zero mean and $\mathbf{Q}_{\mathbf{1}}[n]$ correlation matrix.

In our case, assuming that the state space vector varies randomly around its mean value the state transition matrix is the identity matrix $\mathbf{I}$ of order $M=6$. Furthermore, if the elements of $\mathbf{v}_{\mathbf{1}}[n]$ are taken as independent white noise processes of zero mean and variance $q$ then $\mathbf{Q}_{\mathbf{1}}[n]=q \mathbf{I}$.

- The measurement equation relating the observed signal and the state variables of the system which in matrix form reads:

$$
\mathbf{y}[n]=\mathbf{C}[n] \mathbf{x}[n]+\mathbf{v}_{\mathbf{2}}[n]
$$




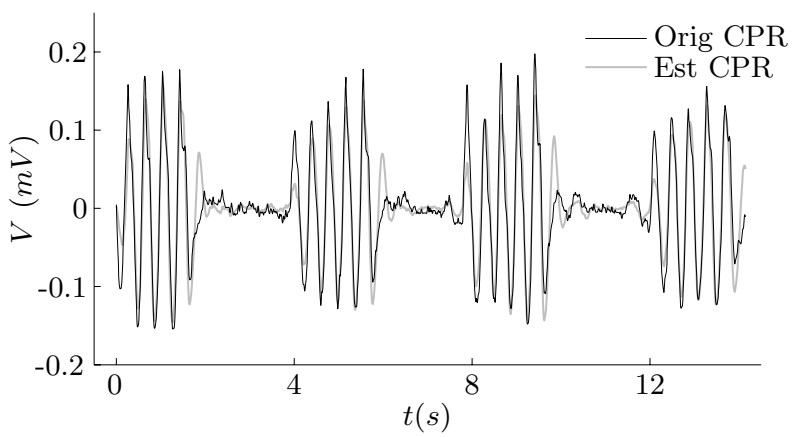

Figure 2. Original and estimated CPR

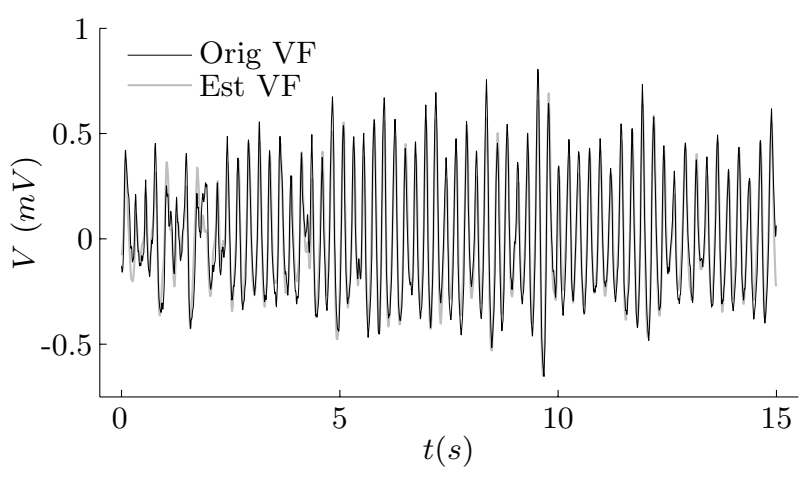

Figure 3. Original and estimated VF
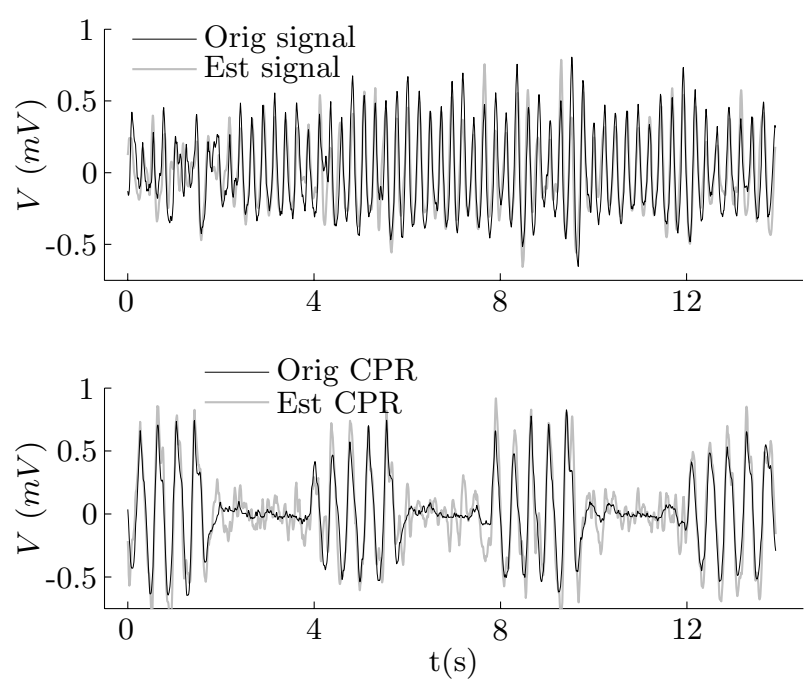

Figure 4. Original and estimated VF and CPR

where $\mathbf{y}[n]_{N \times 1}$ represents the observation vector, $\mathbf{C}[n]_{N \times M}$ the measurement matrix and $\mathbf{v}_{\mathbf{2}}[n]_{N \times 1}$ the measurement noise which is assumed to be zero-mean white noise with correlation matrix $\mathbf{Q}_{\mathbf{2}}[n]$.

In the analyzed case $N=1$ and the observed signal corresponds to the test signal. The measurement matrix is:

$$
\begin{aligned}
\mathbf{C}[n]=\{ & \cos \Omega_{0} n,-\sin \Omega_{0} n, \cos \Omega_{1} n,-\sin \Omega_{1} n, \\
& \left.\cos \Omega_{2} n,-\sin \Omega_{2} n\right\}
\end{aligned}
$$

The measurement noise vector $\mathbf{v}_{\mathbf{2}}[n]$ is then a white noise scalar process of power $Q_{2}$.

The set of recursive equations that solve the Kalman filter [8] produce the estimated VF and CPR signals from the test signal. Figures 2, 3 and 4 show a graphic example of the Kalman filtering scheme described. Figure 2 shows the original and estimated CPR signal in the absence of VF signal, that is for a test signal composed of the interference alone. The estimated and original VF signals in the absence of interference are represented in figure 3 . Both figures demonstrate the rapid convergence of the Kalman filter. An example of the performance for an interference level of $S N R_{i}=0 d B$ showing the original and estimated $\mathrm{VF}$ and CPR signals is provided in figure 4.

\section{Results}

Test signals have been formed for the combination of all CPR and VF registers and three interference levels $\left(S N R_{i}=-6,-3,0 d B\right)$. Two parameters have been used to evaluate the efficiency of the filtering method:

1. The improvement in the signal to noise ratio, $\Delta_{S N R}$ which allows an analytical characterization of the method. A comparison of the signal to noise ratio of the estimated and original VF signals provides the $\Delta_{S N R}$ value.

Figure 5 shows the mean value of $\Delta_{S N R}$ per CPR record for the three $S N R_{i}$. The value strongly depends on the CPR artefact considered. In particular CPR7 produces very poor results due to the important second harmonic component of the artefact. The mean value of the improvement worsens as the $S N R_{i}$ value is increased.

2. The increase in the sensitivity of a AED rhythm analysis algorithm. The sensitivity figures obtained in a commercially available AED (Reanibex 200) for the filtered VF signals gives a practical measure of the goodness of the method. Figure 6 displays the mean sensitivities obtained per CPR for a $S N R_{i}=-3 d B$ before and after filtering. For an artefact free situation the AED gives a sensitivity value of $99.0 \%$. Sensitivities above $90 \%$ have been obtained for all CPR registers but CPR9 (87.4\%).

\section{Discussion and conclusions}

The performance of an adaptive Kalman algorithm for the CPR interference cancellation from the VF signal has been analyzed. A reference signal generated from 


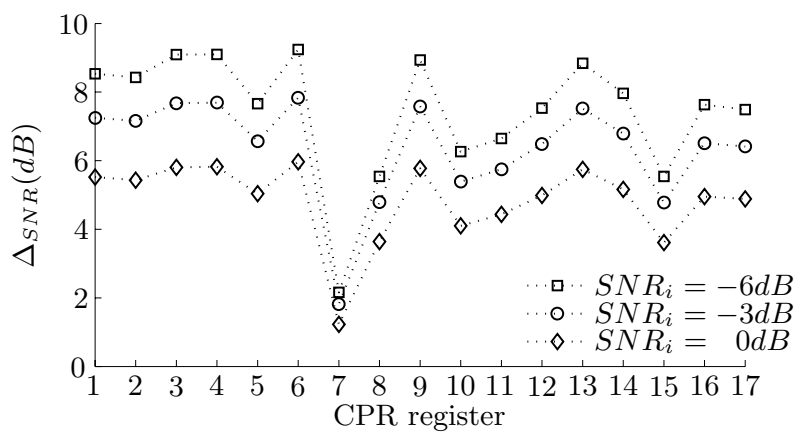

Figure 5. Detailed $\Delta_{S N R}$

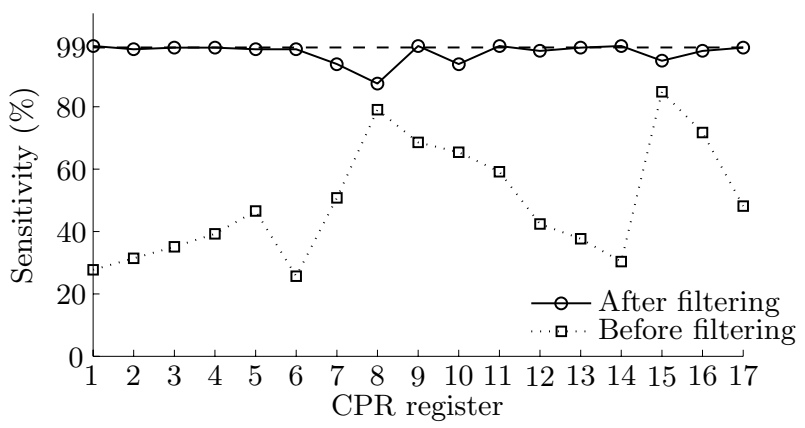

Figure 6. Detailed sensitivity values, $S N R_{i}=-3 d B$

the thoracic compression instants, and strongly correlated to the CPR interference, has been used. This allows an easy estimation of the fundamental frequency of the interference.

The signal to noise ratio improvement obtained is similar to the values reported in other studies $[9,10]$ that rely on the same reference signal. For CPR artefacts showing important second harmonic components the adaptive LMS method described in [10] produces better results than the ones obtained through the proposed Kalman filter.

Regarding the increase in sensitivity of a commercial AED the results obtained through the Kalman filter are substantially better than the ones reported in [10]. This is due to the rapid convergence of the Kalman filter which produces an estimated VF that accurately follows the artefact free VF waveform.

Despite the simplicity of the VF signal model the results obtained are satisfactory and do not strongly depende on the $f_{1}$ and $f_{2}$ values selected. For values of these parameters in the $3.5-6 \mathrm{~Hz}$ range produce comparable results.

Further works should make use of a wider CPR artefact database in order to separate the development from the testing of the algorithm.

\section{Acknowledgements}

The authors would like to thank the support of the MCYT (Spain) through the project TIC2003-08460.

\section{References}

[1] Wik L, Hansen T, Fylling F, et al. Delaying defibrillation to give basic cardiopulmonary resuscitation to patients with out-of-hospital ventricular fibrillation. JAMA 2003; 289:1389-1395.

[2] Cobb L, Fahrenbruch C, Walsh T, et al. Influence of CPR prior to defibrillation in patients with out-of-hospital ventricular fibrillation. JAMA 1999;281:1182-1188.

[3] Strohmenger HU, Lindner KH, Keller A, et al. Spectral analysis of ventricular fibrillation and closed-chest cardiopulmonary resuscitation. Resuscitation 1996; 33:155-161.

[4] Noc M, Weil M, Tang W, et al. Electrocardiographic prediction of the success of cardiac resuscitation. Crit Care Med 1999;27(4):708-714.

[5] Aase S, Eftest $\varnothing 1 \mathrm{~T}$, Hus $\varnothing$ y, et al. CPR artefact removal from human ECG using optimal multichannel filtering. IEEE Trans Biomed Eng 2000;47(11):1440-1449.

[6] Husøy J, Eilevstjønn J, Eftestøl T, et al. Removal of cardiopulmonary resuscitation artefacts from human ECG using an efficient matching pursuit-like algorithm. IEEE Trans Biomed Eng 2002;49(11):1287-1298.

[7] Eilevstjønn J, Eftestøl T, Aase S, et al. Feasibility of shock advice analysis during CPR through removal of CPR artefacts from the human ECG. Resuscitation 2004;61:131141.

[8] Haykin S. Adaptive Filter Theory. 3rd edition. Englewood Cliffs, NJ: Prentice-Hall, 1986.

[9] Aramendi E, Ruiz J, Ruiz de Gauna S, et al. A simple effective filtering method for removing CPR caused artefacts from surface ECG signals. Computers in Cardiology 2005;

[10] Irusta U, Ruiz de Gauna S, Ruiz J, et al. A variable step size LMS algorithm for the suppression of the CPR artefact from a VF signal. Computers in Cardiology 2005;.

Address for correspondence:

Sofia Ruiz de Gauna

School of Engineering

Alameda Urquijo, s/n

E-48013 BILBAO (Spain)

E-mail: sofia.ruizdegauna@ehu.es 